\title{
Editorial \\ Catalysis in Zeolites and Zeotypes-Cornerstone of Chemical Industry and Permanent Subject of Research
}

\author{
Roman Bulánek
}

check for

updates

Citation: Bulánek, R. Catalysis in Zeolites and Zeotypes-Cornerstone of Chemical Industry and Permanent Subject of Research. Catalysts 2022, 12, 53. https://doi.org/10.3390/ catal12010053

Received: 20 December 2021 Accepted: 31 December 2021 Published: 4 January 2022

Publisher's Note: MDPI stays neutral with regard to jurisdictional claims in published maps and institutional affiliations.

Copyright: (C) 2022 by the author. Licensee MDPI, Basel, Switzerland. This article is an open access article distributed under the terms and conditions of the Creative Commons Attribution (CC BY) license (https:// creativecommons.org/licenses/by/ $4.0 /)$.
Department of Physical Chemistry, Faculty of Chemical Technology, University of Pardubice, Studentska 573, 53210 Pardubice, Czech Republic; roman.bulanek@upce.cz

Mankind has been aware of zeolites since 1756, when the Swede, Axel Fredrik Cronstedt (1722-1765), observed the strange behavior of a certain natural mineral (probably stellerite with some amounts of stilbite). It took almost 200 years before zeolites ceased to be minerals that were only on display in museums and became a scientific and commercial success story. It all started with finding a way to prepare zeolites in the laboratory and then using synthetic zeolites in oil refining and petrochemistry. One of the key moments in the history of the industrial use of zeolites was the introduction of the use quaternary ammonium hydroxides (and later other organics) in the synthesis of zeolites, which led to the discovery of a number of new zeolite structures (many of them having no known natural counterparts). The number of members of the zeolite family has steadily increased and now exceeds 250 members. One of the latest synthesis protocols, called ADOR (AssemblyDisassembly-Organization-Reassembly), provides the ability to synthesize isoreticular structures that retain crystalline layers but forms different channel systems and thus new zeolite structures. Very recently, the synthesis of so-called "unfeasible" zeolites (which means zeolite frameworks that do not obey certain rules, especially those related to the correlation between framework energy and density) via the use of the ADOR protocol was demonstrated. This offers the possibility of preparing other zeolites not accessible by traditional solvothermal approach and opens up new, unsuspected horizons for the synthesis of zeolite structures. Zeolites have indelibly entered the history of catalysis. Nowadays, the majority of the basic chemicals and fuels have passed through microporous catalyst-zeolites. The best illustration of the potential of zeolites as catalysts is their application in fluid catalytic cracking, which has led to a complete change in the oil refining industry in the last century. Other examples of the successful use of zeolites as catalysts are $\mathrm{C}_{5-6}$ hydro-isomerization, hydro-cracking, aromatics production and processing, the methanol-to-olefins process, and many others. Discoveries from the last 50 years in zeolite chemistry have led to the establishment of a new branch in material chemistry and new concepts in catalysis. Against this background, zeolites have excellent results and exhibit all of the required properties to be in an excellent position for further growth in industrial applications. The articles collected in this Special Issue focus on new trends in zeolite synthesis and their application in the catalytic conversion of various feedstocks into valuable chemicals.

Two review articles offer interesting overviews of hot topics in the advanced synthesis of zeolites. Mordenite is one of the most important strongly acidic zeolitic catalyst that is widely used in industry in various processes including alkylations, isomerizations, dehydration, and aminations. The slow diffusion of reactants or products through the catalyst particles often plays an important role in these processes. The current trends in solving this problem are efforts to synthesize catalysts in the sub-micrometer or nanometer dimensions and the generation of hierarchical micro-mesoporosity in these zeolites. The review by Kalvachev et al. [1] describes recent advances in the synthesis of nanosized and hierarchical mordenite materials. The first part of this review attempts to fine-tune and control particle size through the conditions of solvothermal synthesis. The second part of the review focuses on the dependence of the texture (especially the pore size distribution) 
of post-synthetically treated mordenites on the conditions of alkali and acid treatments. In line with the requirements for more economical and environmentally sustainable zeolite production, new synthesis processes with a lower environmental impact and a reduction in waste are intensively sought. Kornas et al. [2] summarizes the state of the art of the solvent-free synthesis of zeolites by mechanochemical activation in their review. Solventfree strategies have attracted great attention in last decade due to high zeolite yields, water consumption minimization, and thus wastes reduction and energy efficiency. The possibilities and limitations of milling-based activation methods are discussed and are highlighted through numerous practical examples. Each practical example is accompanied by an analysis of the conditions and a detailed characterization of the prepared materials. The article is thus a real practical guide for all those who are interested in this synthetic procedure and who want to implement it into their practice.

The direct conversion of methane to methanol is great challenge. Metal-functionalized zeolites are the most promising catalysts for this process. One of the biggest obstacles in the implementation of this catalytic process in industry is the problems that are related to the extraction of methanol from the active center of the catalyst. Difficult desorption leads to over-oxidation to $\mathrm{CO}_{2}$ or to the formation of dimethyl ether. Therefore, understanding these effects on the affinity between methanol and the catalyst surface is crucial to the development of an effective catalyst. Creci et al. [3] studied the effect of the nature of heteroatoms in the MFI framework on the methanol-zeolite interaction energies and its desorption rates. They conclude that the lower the acidity of the zeolite, the easier it is for methanol to be desorbed from the surface. Methanol is usually used as a starting material for other processes. One industrially relevant process is the conversion of methanol to hydrocarbons (MTH). This process operates via the so-called dual cycle hydrocarbon pool mechanism, which requires a certain amount hydrocarbon species to be present in the micropore to function. In their paper, Parker and Kombanal [4] report a theoretical study on the calculation of the volume required for various compounds that must be present in the working catalysts. The study shows that size and shape of the zeolitic micropores play a decisive role in determining the nature of the molecules causing the deactivation. The results reported in this paper clarify why MFI zeolites exhibit exceptional performance in the MTH reaction.

The next two articles also deal with the issue of the deactivation of zeolitic catalysts in various processes. The study by Sajad et al. [5] is devoted to the detailed research of the activity of a composite material that consists of a La-FAU zeolite supporting a eutectic mixture of $\mathrm{KCl}$ and $\mathrm{MgCl}_{2}$ as a catalyst for the oxidative dehydrogenation (ODH) of ethane to ethene. This molten salt-like catalytic system exhibits very high activity and selectivity in ethane $\mathrm{ODH}$, but at the same time, it also exhibits very fast deactivation. Through a detailed characterization of the spent catalyst as a function of the time-on-stream and an analysis of the reaction products by mass spectrometry, the loss of catalytic activity was associated with the loss of chlorine from molten salts to form chlorinated hydrocarbons such as chloromethane, ethyl chloride, and chloroethene. In the last paper, Stellato et al. [6] investigated the cracking of 4-propylphenol (a model compound for lignin monomer) over MFI zeolite. This zeolite deactivates due to the chemisorption of the phenolates on Lewis acid sites and by hindering diffusion through the zeolite channel. This deactivation can be suppressed by co-feeding water, which facilitates the removal of the phenolates from the catalyst through hydrolysis. The multi-spectroscopic characterization of the spent catalysts revealed the secondary, slower but more permanent, deactivation process that is associated with the slow deposition of polyaromatic species that are produced from light olefins as the by-product of lignin cracking.

In conclusion, this collection of publications is a good representation of the recent topics and latest trends in zeolite chemistry and catalysis. I wish to thank the authors for their valuable contributions, and I hope that this Special Issue will be inspiring for the many scholars who are active in this field. 
Funding: This research received no external funding.

Conflicts of Interest: The author declares no conflict of interest.

\section{References}

1. Kalvachev, Y.; Todorova, T.; Popov, C. Recent Progress in Synthesis and Application of Nanosized and Hierarchical Mordenite-A Short Review. Catalysts 2021, 11, 308. [CrossRef]

2. Kornas, A.; Olszówka, J.E.; Klein, P.; Pashkova, V. Milling Activation for the Solvent-Free Synthesis of Zeolites. A Practical Guide. Catalysts 2021, 11, 246. [CrossRef]

3. Creci, S.; Martinelli, A.; Vavra, S.; Carlsson, P.-A.; Skoglundh, M. Acidity as Descriptor for Methanol Desorption in B-, Ga- and Ti-MFI Zeotypes. Catalysts 2021, 11, 97. [CrossRef]

4. Parker, S.F.; Kombanal, A.J. How Many Molecules Can Fit in a Zeolite Pore? Implications for the Hydrocarbon Pool Mechanism of the Methanol-to-Hydrocarbons Process. Catalysts 2021, 11, 1204. [CrossRef]

5. Sajad, M.; Bulánek, R.; Šlang, S. Physico-Chemical Changes in the $\mathrm{KCl}-\mathrm{MgCl}_{2}$ /La-FAU Composite Catalyst Induced by Oxidative Dehydrogenation of Ethane. Catalysts 2021, 11, 392. [CrossRef]

6. Stellato, M.J.; Innocenti, G.; Bommarius, A.S.; Sievers, C. Pore Blocking by Phenolates as Deactivation Path during the Cracking of 4-Propylphenol over ZSM-5. Catalysts 2021, 11, 721. [CrossRef] 